\title{
From nano machines to Nobel prizes
}

\author{
Chris Toumey ponders the nanotechnology behind the 2016 Nobel Prize in Chemistry.
}

In November 2003, scientists at Cornell University played a chord on the world's smallest musical instrument, a guitar ten micrometres long. Dustin Carr and Harold Craighead used photolithography to etch this device onto a silicon surface so that it had six strings, each 50 nanometres in diameter. To make the strings produce noise, they strummed them with the ultrafine laser of an atomic force microscope ${ }^{1}$.

Unfortunately, it was impossible for anyone to hear the sound. The chord was seventeen octaves higher than the highest octave that the human sense of hearing can detect.

What then was the point of the nano guitar? At the time it was speculated that artefacts like this could be "extremely sensitive detectors for ultra-high frequency waves”, but this has not happened, as best as I can tell. The tiny guitar was a one-off accomplishment that led to nothing else (if I am wrong about this, I would be happy to learn what resulted from playing that chord).

There is nothing wrong with having fun in the Cornell Nanoscale Science and Technology Facility, nor with showing that nanotech does clever things. Even so, it is fair to ask whether the science is really going to change our lives, and if so, how?

This brings us to the nano elevator. Not long after we heard about the nano guitar, we learned of a device $2.5 \mathrm{~nm}$ high which moved $0.7 \mathrm{~nm}$ up and down ${ }^{2}$. Sir Fraser Stoddart, Alberto Credi and their teams at UCLA and the University of Bologna caused these devices to assemble themselves. Exposure to a base made it move down, and to an acid made it move up.

I know what you're thinking: if this elevator moves less than a nanometre, then wouldn't it be quicker to take the stairs? But in fact it has a value more tangible than that of the nano guitar: the nano elevator can encase a drug molecule and then open up to release the drug when it gets to where the drug will act on the cause of an illness. In other words, an efficient drug delivery device.

Another such device came from the lab of David Gracias at Johns Hopkins University. Also using photolithography, Gracias and his team cut out two-dimensional patterns that folded into three-dimensional cubes. Each side was 100 to $200 \mathrm{~nm}$ in length and width. The sides had large or small openings, depending on which reagents they were meant to deliver.
Magnetic fields steered these metallic cubes to where they were needed, and the reagents then escaped from the cubes. Thus another vehicle for precisely delivering and releasing drugs ${ }^{3}$.

This brings us to rotaxanes and catenanes. During 1978-1981, Fraser Stoddart began work on mechanical bonds in chemistry. Later (1988-1991), this led to the development of molecular structures that could be assembled "like a rod through a ring" or like beads on an abacus. These are called rotaxanes, and they are described as "a molecular shuttle" and as "linear molecular motors powered by chemicals, electricity and light" 4 . The nano elevator is a sophisticated rotaxane: it moves up and down within a confined space such that its movement can be controlled by dosing it with bases or acids.

Meanwhile, Jean-Pierre Sauvage was creating catenanes at the University of Strasbourg. These are interlocking synthetic molecular rings which alternate between equilibrium states and energy states ${ }^{5}$. They are important because they can serve as on/off switches in memory devices.

The third piece of this story is the work of Bernard Feringa at the University of Groningen. Feringa first created a molecular motor, and later invented a nano car, with four wheels and a chassis.

Here a personal note: about 10 years ago, I discovered a computer game called Nano Quest. This was created by the Centre for Research on Adaptive Nanostructures and Nanodevices and others at Trinity College Dublin. Its premise is that a student of nanotechnology has been shrunk down to the nanoscale, and then needs to complete a series of tasks to return to the human scale. The student encounters carbon nanotubes and $\mathrm{C}_{60}$ while travelling across graphene and other atomic surfaces.

The first task is to assemble three nanotubes and four $\mathrm{C}_{60}$ spheres to make a nano car and then drive it to places where the players encounter more nanoscale challenges. The one in Nano Quest was based on a vehicle created by James Tour and his team at Rice University, but I like to imagine that Bernard Feringa is the back-seat driver instructing the student how to operate the nano car.

It took me weeks to get good at Nano Quest, and then it pleased me to introduce it to high school students in our summer programmes on nanotechnology. I did not notice that my son was quickly mastering Nano Quest, beginning with the nano car. Before long he was so proficient that I started playing only when he was not around. I recommend Nano Quest as a fun way to learn about the nanoscale, but, remember, our children will embarrass us at it!

Back to rotaxane, catenane and nano cars: the research on these controllable nanoscale devices began to appear in scientific journals in the 1990s and continued into the next decade. Thus, nanotechnology achieved a credible reputation for being able to control matter and fabricate gadgets at remarkably small sizes. I say 'control' and 'fabricate' for a reason. Nanotech includes understanding matter, but it is much more than scientific understanding. It endeavours to improve our material culture by making new material realities that are better than what we have now.

The 2016 Nobel Prize in Chemistry went to Stoddart, Sauvage and Feringa for showing that their nanoscale machines can lead to improved drug delivery, improved microelectronics, and improved control of nanoscale matter. We are not talking about amusing toys. We are talking about enhancing important features of the world we inhabit so that our lives become better.

Although the nano guitar may not count, I still want someone to invent an amplifier or whatever it takes for us to hear that chord. Maybe it will remind us of iconic chords from the Beatles, the Stones, or other bands from my teenage years. But even if I do not get that satisfaction, I can see why the Nobel Committee for Chemistry chose Stoddart, Sauvage and Feringa. Good choice indeed.

Chris Toumey is in the Center for Environmental Nanoscience \& Risk, Arnold School of Public Health, University of South Carolina, Columbia, South Carolina 29208, USA.

e-mail:Toumey@mailbox.sc.edu

\section{References}

1. Johnson, G. Ideas \& trends: Atomic scales; striking notes of progress on the world's tiniest guitar. New York Times (9 November 2003).

2. Fountain, H. Mr. Otis, call your office: A nano-elevator is built. New York Times (23 March 2004).

3. Service, R. Science 313, 1032-1033 (2006).

4. Saxl, O. Knight of the nano-realm. Nano Now (February 2007); http://go.nature.com/2h1Exq5

5. UCLA's J. Fraser Stoddart on switching to molecular electronics. Science Watch (September 2005); http://go.nature.com/2h1BYED

Corrected after print: 19 January 2017 


\section{Correction}

The Thesis 'From nano machines to Nobel prizes' (Nat. Nanotech. 12, 1; 2017) has been updated to more clearly explain some aspects of the early development of the mechanical bond 